\title{
Designing disorder at the atomic scale
}

The crystal structures of solids can exhibit a vast array of different atomic arrangements, which may also change depending on conditions,

temperature, pressure, etc. In Dr Facility (ESRE) in France have used cutting-edge techniques to show for the first time how some materials display exciting new properties when they are preferred atomic arrangements. Their results could pave the way for many useful applications, influence on the ways in which physicists view disorder within solid materials. Dr research, a team led by prevented from adopting their

he orderly arrangement of atoms
or molecules on a 'crystal lattice' is a common feature of many solid materials. When seeking to understand the physical properties of a material, and to predict how it will behave in different scenarios, it is crucial for physicists to precisely as possibe To do this, studies carried out over the past centur studies measured how beams of radiation, most often $x$-rays, are scattered as they pass through crystals.

'Classical crystallography' techniques allow researchers to accurately measure the spacings between the atoms as they are arranged on the lattice - enabling them to successfully predict a wide variety of material properties. Yet as modern crystallography techniques become more and more sensitive, physicists are increasingly finding that the crystal structures of many solids are less orderly than once thought. In some cases, certain atoms can be shifted in unexpected positions, replaced with oth ypes of atoms, or even removed

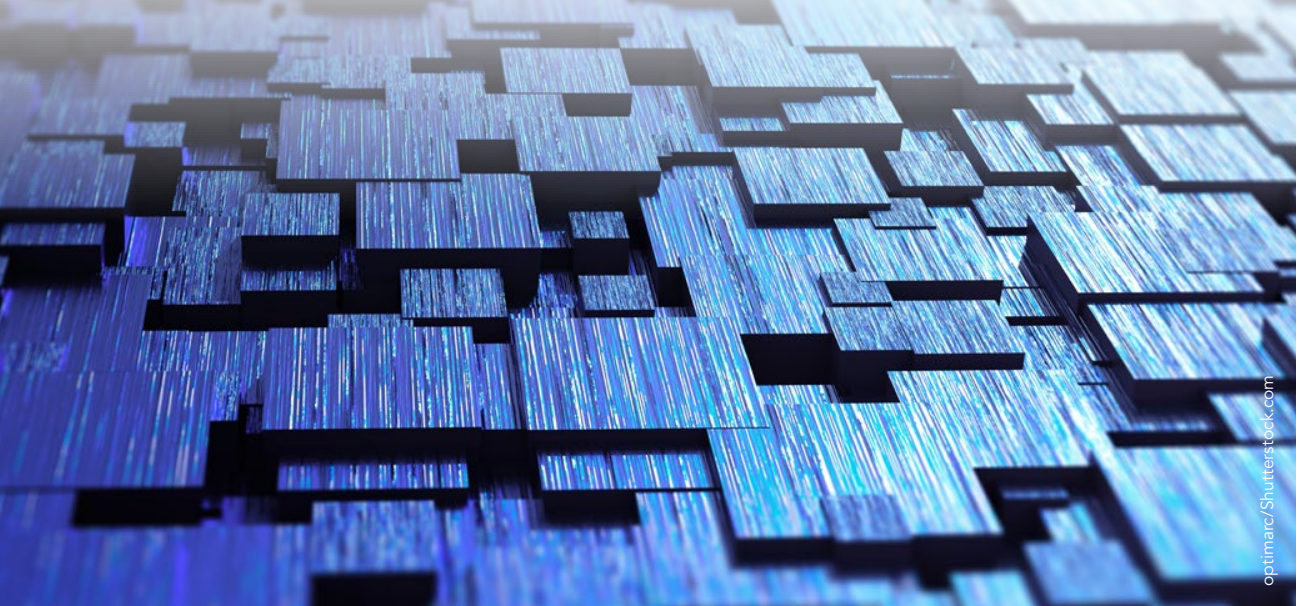

Furthermore, these features aren't always random: in some materials, clear patterns can emerge, giving rise to short-range correlations between disordered atoms. For Dr Chaney (European Synchrotron Radiation Facility (ESRF), France), this consider. 'This for materials physicists to "correlted disorde" anon is known as for many of the exotic properties found in promising new materils' he explains. Through their research. DrChaney's team have revealed an entirely new form of correlated disorder, which could have important implications for our understanding of how solid material behave and may be exploited when designing future advanced materials.

\section{INDUCING POSITIONAL DISORDER} Crystals can display a diverse array of atomic geometries: from simple cubic lattices, to more complex hexagonal patterns and even arrangements with far less symmetry such as the orthorhombic sructures. However, tin many cases these vangen and pressure, materials will always seek to possess the lest amount of energy possible, by transitioning between different atomic arrangements.

In their latest study, Dr Chaney's team, including researchers from the University of Bristol, UK, focused on a particular effect named 'positional' disorder: describing points within a lattice where toms can be found at some distance away from their expected positions.

To study this phenomenon, the esearchers turned to a technique called 'epitaxial deposition', which allows for hin crystal films to be built up layer-by that the atomic structure of the film

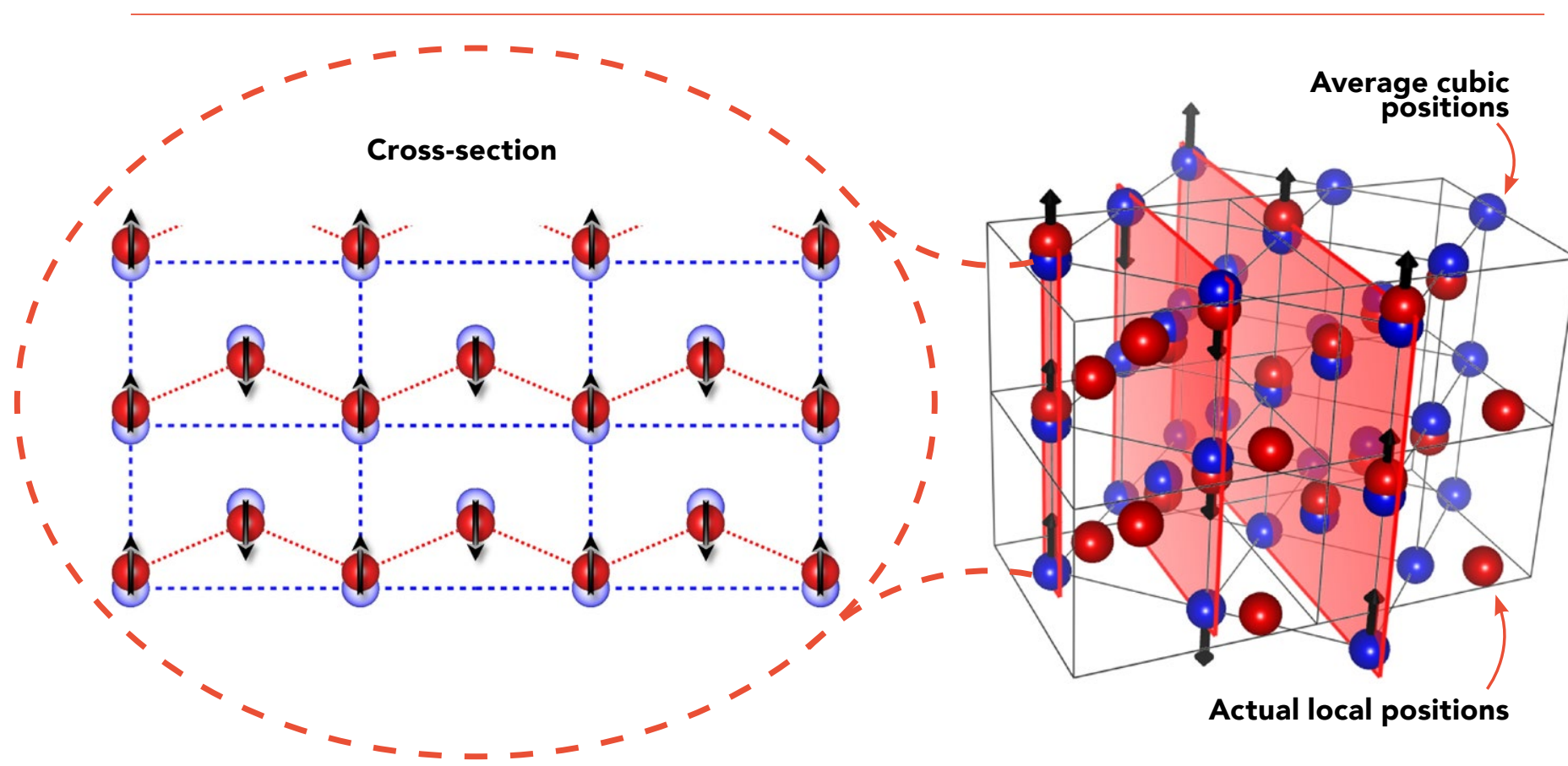

Figure 1. Right: The relationship between the average, cubic crystal structure (blue) and the actual, local crystal structure (red). To transition from
the average to local structure the atoms within each red plane move together in the direction indicated by black arrows. Note that only three select the average to local structure the atoms within each red plane move together in the direction indicated by black arrows. Note that only three select
planes are shown but every atom is displaced, Left: a cross-section perpendicular to the red planes highlighting the difference in atomic positions.

has a well-defined relationship to that of the substrate. More specifically, the team used a method named 'epitaxial matching.' Dr Chaney describes, 'with this technique, two different crystals with as the metal cools. In this case, however, the picture changed entirely.

motion of the ball can fall into small similar structures can be grown atop one the UMo alloy, it is first important to will continue rolling down the hillside, $\begin{array}{ll}\text { another, such that } & \text { until it reaches } \\ \text { they lock together } & \text { the bottom. }\end{array}$

like jigsaw pieces.' As a form of disorder that has gone This interlocking undetected, this phenomenon could

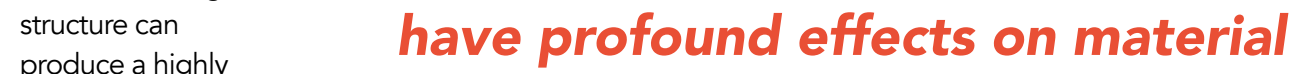
produce a highly
unique property:

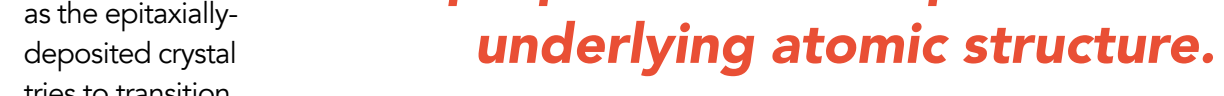
tries to tra

In this case, the rolling of the ball is comparable to a cooling alloy's continually changing crystal structure, and the smaller valleys on

from one form into another, sometim ' 'substrate below Such that 'f of of the subsequently tries to adopt a different structure, this interlocking can frustrate the transition,' Dr Chaney explains.

grasp the concept of 'metastability' in think of a ball rolling down a roughlytextured hill, pulled downward by gravity In some cases, the downward

tomic arrangements way. Although these structures may be stable momentarily, they will inevitably decompose after a certain time, until the material reaches its lowest energy state.

The team used these epitaxial matching techniques to construct an alloy containing a mixture of uranium and molybdenum (UMo), on top of a niobium substrate. Measuring just 300 nanometres thick, the material was deposited at temperatures of $800^{\circ} \mathrm{C}$. Under these conditions, the uranium and molybdenum atoms are arranged would typicaly reorcan into another lower symise try structes

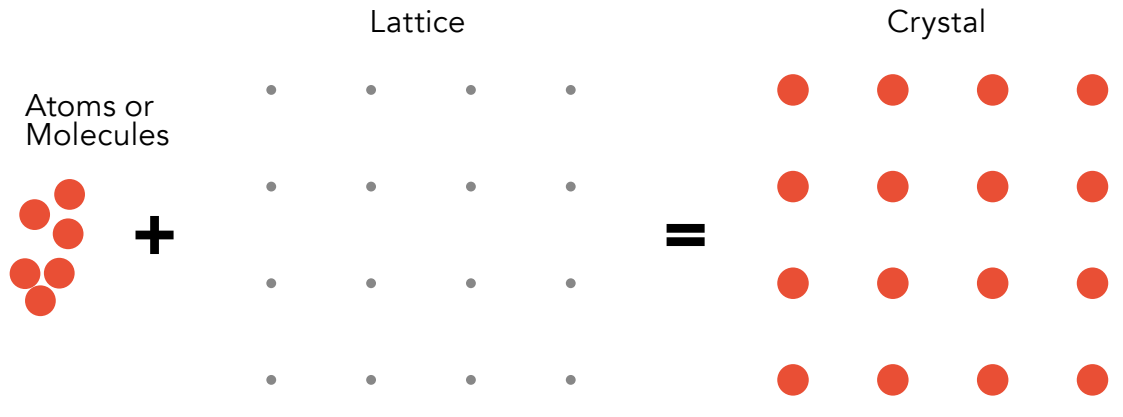

Figure 2. A crystal structure is formed when an atom, group of atoms
added to every lattice position. 


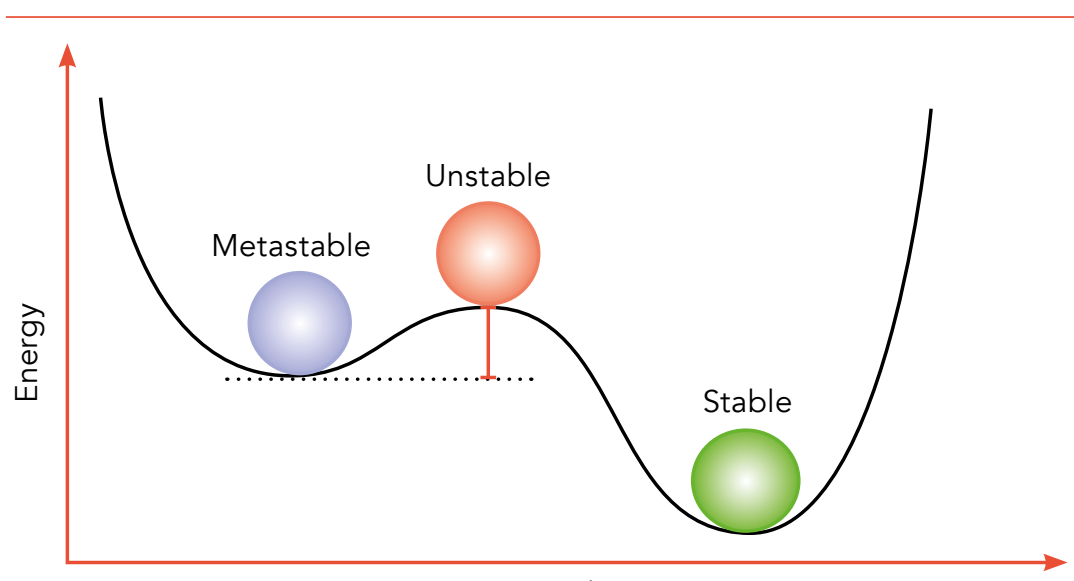

Coordinate

Figure 3. A general 'hill and valley' energy diagram showing stable, unstable and metastable
states. The orange bar indicates the energy needed to move from the metastable to stable state.

As a result, these temporary valleys of stability are named 'metastable' states.

As Dr Chaney discovered, if a UM lloy is epitaxially matched with between metastable states are prohibited from occurring - as if ball had permanently come to $r e s$ in a small hillside valley. 'Instead of decomposing, interlocking forces the UMo alloy to retain its cubic structure as it cools, preventing uranium from adopting its preferred orthorhombic crystal structure,' he explains. As whole, the atomic arrangement remains far more symmetrical than the alloy's uranium atoms would prefer

\section{A NEW FORM OF DISORDER}

Thudy the characteristics of their new metastable material in more the scattering of $x$-ray beams as they passed through the film. In itself, this cystallography technique presented significant challenge: while x-ray scattering experiments are now very common, thin films are difficult to study since there is little material available to scatter the beam. Fortunately, the x-ray beam at ESRF was intense enough to overcome this challenge, allowing Dr Chaney and his colleagues to clearly dentify the atomic arrangement of their alloy, despite the film thickness being ust $1 / 70$ th the diameter of an average

Remarkably, their results revealed disorder. While the symmetry of the
These efforts revealed that the correlated disorder, which exists only to large changes in the materil's physical properties. 'By combining th scattering experiments with theoretical simulations, we showed that this exotic form of disorder strongly affects how the atoms vibrate, ' Dr Chaney illustrates. 'In turn, it dramatically reduces the effectiveness with which these vibration can carry thermal energy.'

\section{DESIGNING FUNCTIONALITY}

Overall, the correlated disorder

uncovered by the team arises from a 'crystallographic conflict' between the preferred, atomic-scale symmetry of atoms within the alloy, and the higher symmetry of the lattice they have been arranged upon. Importantly, these alloy they studied, and ins the specific be expected in any material where such 'crystallographic conflict' can be produced. As a form of disorder that has gone entirely undetected so far, this phenomenon could have profound effects on any material properties that depend on the underlying atomic structure. 'This makes it an exciting tool for designing disorder and functionality into materials, and hints at a deep link

Remarkably, their results revealed an entirely new type of correlated disorder.

Now, there is an oft-quoted adage amongst physicists that 'structure drives function', that is, many of a materi underlying arrangement of its atoms. Given this, the team set about to explore whether the correlated disorder evident on an atomic scale would manifest into an observable change in the macroscopic properties of the material. More specifically they used a technique called 'inelastic $x$-ray scattering' to probe how vibrations travel through the lattice. To further strengthen their results, Dr Chaney's team supplemented their experimental investigations with computer models based on the most advanced physical theories to date. These can make predictions of crystal structures in varying environmental vibrational properties. etween metastability and correlated disorder' Dr Chaney concludes.

On one level, this success presents promising opportunities for physicists to engineer new, tuneable, and highly exotic materials - which could conduct heat and electrical current in unusual, and potentially incredibly useful new the implications of his team's results could go deeper: arguing that the ability of a material to host correlated disorder could be the very property that allows many metastable systems to exist in the first place. If correct, this may call for physicists to take a closer look at many materials previously considered well understood and instead go hunting for previously thought. unusual forms of disorder which may

\section{Behind the Research}

\section{Dr Daniel Chaney}

E: daniel.chaney@esrf.fr T: +447813802531 W: www.researchgate.net/profile/Daniel-Chaney-4

\section{Research Objectives}

Dr Daniel Chaney researches new behaviours arising from which to control such disorder.

Detail

AddFF, 71 Avenue des Martyrs, 38043 Grenoble, France

Daniel attended the University of Bristol, UK, from 2012 to 2021 , first obtaining a masters degree in physics in 2016 before completing his PhD in 2021. He is currently in Grenoble, France, where he holds a postdoctoral scientist Radiation Facility (ESRF).

Funding

- Engineering and Physical Sciences Research Council - PhD studentship (ICase Award)

-PhD studentship (ICase Award : Industrial Sponsor

-European Synchrotron Radiation Facility - six-month taineeship contract and beamtime

The researchers note the following copyright: UK Ministry of Defence $\odot$ Crown Owned Copyright 2022/AWE.

\section{Collaborators}

Sastellano - Le Commissariat à l'énergie atomique et aux énergies alternatives

European Synchrotron

Radiation Facility et aux énergies alternatives

ariat à lénergie atomique et aux energies alternatives

Boris Dorado - Le Commissariat a l'energie atomique et

aux energies alternatives

Luigi Paolasini - The European Synchrotron

Radiation Facility

Sophie Rennie - University of Bristol

- Christopher Bell - University of Bristo

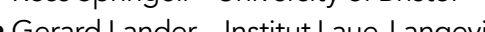

- Gerard Lander-Instiversity of Bristol

\section{References}

Chaney, D, Castellano, A, Bosak, A, et al, (2021) Tuneable correlated disorder in alloys. Physical Review Materials, $5(3)$,
035004. doi.org/10.1103/PhysRevMaterials.5.035004 Castellano, A, Bottin, F, Dorado, B, Bouchet, J, (2020) Thermodynamic stabilization of $\mathrm{Y}$-U-Mo alloys: Effect of Mo content and temperature. Phys
org/10.1103/PhysRevB.101.184111

Simonov, A, Goodwin, AL, (2020) Designing disorder into crystalline materials. Nature Reviews Chemistry, $4(12)$

Keen, DA, Goodwin, AL, (2015) The crystallography of correlated disorder. Nature, 521(7552), 303-309. doi. org/10.1038/nature 14453

\section{Personal Response}

\section{What opportunities could be presented by inducing
correlated disorder in other materials?}

II The most obvious avenue of exploration would be designing new types of materials within the class interesting materials that generate electricity from a temperature gradient and vice versa. Importantly, the key factor in their efficiency is the ratio between a material's electrical conductivity and its thermal form of correlated disorder can dramatically red thermal conductivity mediated by atomic vibrations however, as a metallic alloy the majority of the thermal conductivity in our samples is unfortunately carried by the electrons. If instead one could realise the same type of disorder in a semiconducting or insulating materie thermoelectric materials. 